\title{
SIMULAÇÃO DO EFEITO ANTINOCICEPTIVO DE DETOMIDINA EM CAVALOS
}

\author{
Marco Aurélio Camargo Fontanela ${ }^{1}$; Marcos Ferrante ${ }^{2}$
}

1 Residente em Anestesiologia Veterinária, Depto. Medicina veterinária, Universidade Federal de Paraná (UFPR), Curitiba, PR - Brasil.

2 Prof. de Farmacologia Veterinária, Depto. Medicina veterinária, Universidade Federal de Lavras (UFLA), Lavras, MG - Brasil (marcosferrante@gmail.com)

Recebido em: 06/04/2019 - Aprovado em: 10/06/2019 - Publicado em: 30/06/2019 DOI: 10.18677/EnciBio_2019A66

\section{RESUMO}

Este estudo teve por objetivo simular o efeito antinociceptivo de detomidina por via IV e IM em cavalos na dose de $80 \mathrm{ug} / \mathrm{kg}$. As analises farmacocinético/farmacodinâmico foi realizado com a equação de.Os dados de farmacocinética e farmacodinâmica foram os correspondentes ao estudo nas doses de $30 \mathrm{ug} / \mathrm{kg}$. O efeito antinociceptivo foi considerado a partir das alterações no limiar nociceptivo. Finalmente, foi realizada determinado o efeito antinociceptivo a partir das doses elevadas de detomidina $(80 \mathrm{ug} / \mathrm{kg})$ a partir das concentrações plasmáticas mediante uma a simulação. O efeito antinociceptivo simulado a partir das concentrações plasmáticas de correspondentes a doses de $80 \mathrm{ug} / \mathrm{kg}$ foi de $90 \%$, $80 \%$ e $40 \%$ até 120, 180 e 240 minutos para a via IM e foi de $90 \%$, 80\% e $60 \%$ até, 120, 180 e 240 minutos para a via IV, respectivamente. Doses elevadas de detomidina determinam efeitos superiores e mais douradores respeito das doses de $30 \mathrm{ug} / \mathrm{kg}$ pelas respectivas vias de administração. Porém, são necessários realizar estudos de in vivo completares nessas doses elevadas que verifiquem os efeitos adversos.

PALAVRAS- CHAVE: Analises PK/PD, analgésicos, determinação de doses.

\section{DETOMIDINE ANTI-SOCIETY EFFECTS SIMULATION STUDY IN HORSES}

\section{ABSTRACT}

This study aimed to simulate the antinociceptive effect of detomidine IV and IM in horses at a dose of $80 \mathrm{ug} / \mathrm{kg}$. The pharmacokinetic / pharmacodynamic analysis was performed using the equation. Pharmacokinetic and pharmacodynamic data were those corresponding to the study at doses of $30 æ \mathrm{~g} / \mathrm{kg}$. The antinociceptive effect was considered from the nociceptive threshold changes. Finally, the antinociceptive effect was determined from the high doses of detomidine $(80 \mathrm{ug} / \mathrm{kg})$ from the plasma concentrations by means of a simulation. The simulated antinociceptive effect from plasma concentrations corresponding to doses of $80 \mathrm{ug} /$ $\mathrm{kg}$ was $90 \%, 80 \%$ and $40 \%$ up to 120,180 and 240 minutes for the IM route and was $90 \%, 80 \%$ and $60 \%$ up to 120,180 and 240 minutes for the IV route, respectively. Elevated doses of detomidine determine superior and more gilding effects at doses of $30 æ g / \mathrm{kg}$ by respective routes of administration. However, in vivo studies complete at these high doses are required to verify adverse effects.

KEYWORDS: PK / PD analyzes, analgesics, determination of doses. 


\section{INTRODUÇÃO}

O controle da dor é essencial para bem-estar em diversas situações fisiopatológicas (ELFENBEIN et al., 2014). No entanto, ainda existe um déficit muito grande na medicina de equinos em relação ao controle analgésico, o que se dá possivelmente pela dificuldade de reconhecimento e pela elevada preocupação com os efeitos adversos farmacológicos (LOPES et al.,2017). Os analgésicos utilizados com maior frequência em equinos incluem os anti-inflamatórios não esteroidais (AINEs), opioides e os agonistas alfa 2-adrenérgicos. A detomidina (DET) é um alfa 2 agonista que apresenta potência superior aos fármacos mais antigos como a xilazina. Seu uso em cavalos é principalmente como sedativo, relaxante muscular (GONZALLO-MARCILLA et al., 2017; RANKIN, 2017; GONZALLO- MARCILLA et al., 2018b).

Os modelos farmacocinéticos/farmacodinâmicos (PK/PD) são baseados em determinar a concentração plasmática do fármaco e de maneira simultânea determinar os efeitos causados por essas concentrações. Assim, mediante o modelamento PK/PD é possível estabelecer a correlação que permitirá estimar o efeito em função da concentração plasmática a fim de direcionar experimentações (GONZALLO- MARCILLA et al., 2018a). Por tanto, o conhecimento do perfil farmacocinética (PK) permite a adequação da dosagem com o objetivo de ajustar a concentração do fármaco no sitio de ação. Por outro lado, a compreensão a farmacodinâmica (PD) permite a administração do fármaco segundo as necessidades especificas do animal (BILLARD, 2015).

Existem dois estudos de farmacocinética de DET administrada intramuscular (IM) e intravenosa (IV) em cavalos, o primeiro, realizado por Mama et al. (2009) na dose de $30 \mathrm{ug} / \mathrm{kg}$ e o segundo realizado por Salonen et al. (1989) na dose de 80 $\mathrm{ug} / \mathrm{kg}$. Porém, só no estudo com a dose menor foi determinado o efeito antinociceptivo. Por tanto, este estudo teve por objetivo simular o efeito antinociceptivo de DET por via IV e IM em cavalos na dose de $80 \mathrm{ug} / \mathrm{kg}$.

\section{MATERIAIS E MÉTODOS}

A análise farmacocinético/farmacodinâmico foi realizado com a equação de Hill (Billard, 2015) que permite estabelecer a correlação entre a concentração de DET no plasma e o efeito antinocicetivo (FIGURAS 1 e 2). Os dados de farmacocinética e farmacodinâmica foram os correspondentes ao estudo nas doses de $30 \mathrm{ug} / \mathrm{kg}$ realizado por Mama et al. (2009). O efeito antinociceptivo foi considerado a partir das alterações no limiar nociceptivo.

Finalmente, foi realizada determinado o efeito antinociceptivo a partir das doses elevadas de DET (80 ug/kg) a partir das concentrações plasmáticas mediante uma a simulação (SOLONEN et al. 1989). 


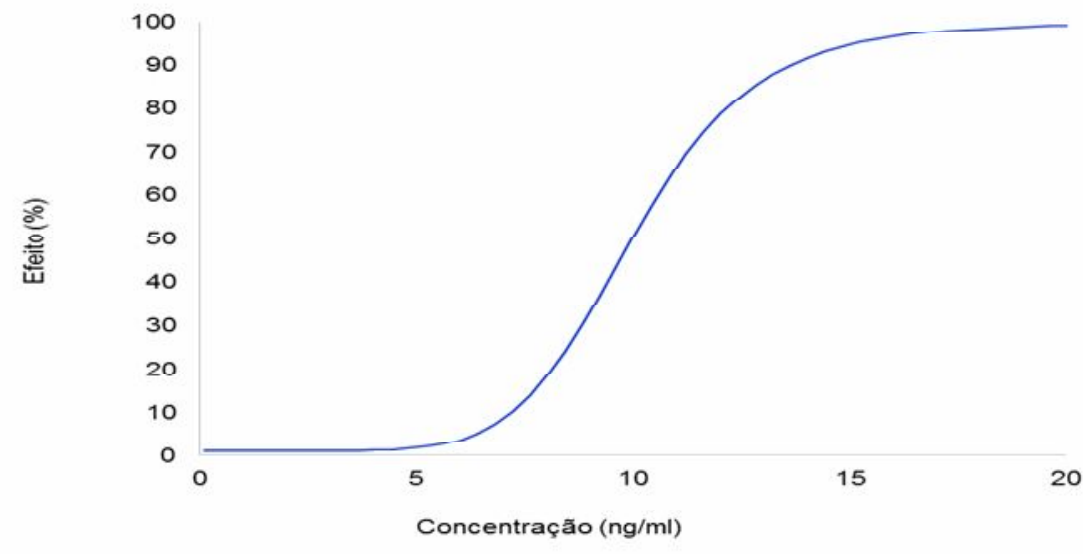

FIGURA 1. Curva da relação entre a concentração e o efeito FONTE: Billard, et al., (2015).

$$
\mathrm{E}=\mathrm{E}_{0}+\mathrm{E}_{\text {max }} \mathrm{x} \frac{\mathrm{C}^{\gamma}}{\mathrm{C} 50^{\gamma}+\mathrm{C}^{\gamma}}
$$

FIGURA 2. Equação de Hill. Onde "E0" o efeito inicial, "E" indica o efeito estimado, "E max X" o efeito máximo, "C50" a concentração para o 50\% do efeito, "C" a concentração, e " $Y$ " o coeficiente de Hill. FONTE: Billard, et al.,( 2015).

\section{RESULTADOS}

As curvas de disposição plasmática de DET após da administração intramuscular e intravenosa de $30 \mathrm{ug} / \mathrm{kg}$ e $80 \mathrm{ug} / \mathrm{kg}$ são apresentadas na figura 3. Enquanto que na figura 4 pode-se observar a relação da concentração no plasma e o efeito antinociceptivo. Finalmente nas figuras 5 e 6 são apresentados os efeitos antinociceptivos simulados correspondentes as doses baixas e elevadas de DET para ambas vias de administração (IV e IM). 


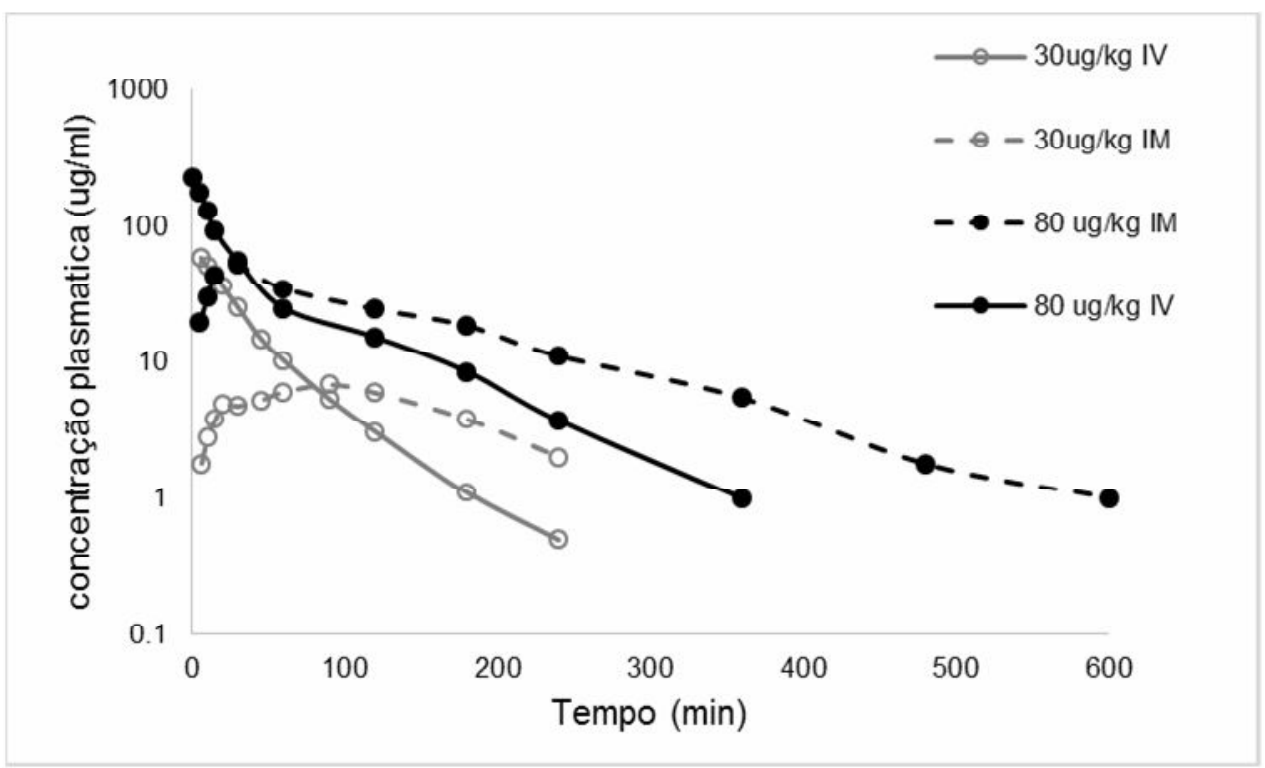

FIGURA 3. Curva de concentração plasmática de DET em cavalos.

FONTE: Solonen et al.,(1989) e Mama et al,. (2009)

Esta simulação determinou que as doses de $80 \mathrm{ug} / \mathrm{kg}$ determinaram efeitos antinociceptivos mais elevados e por maior tempo que as doses de $30 \mathrm{ug} / \mathrm{kg}$ (FIGURAS 3, 5 e 6).

Salonen et al. (1989), utilizando doses de $80 \mathrm{ug} / \mathrm{kg}$ via IM, conseguiu concentrações plasmáticas de $20 \mathrm{ng} / \mathrm{mL}$ cinco minutos após a administração, com um pico de $50 \mathrm{ng} / \mathrm{mL}$, após aproximadamente 30 minutos após administração e concentrações de $20 \mathrm{ng} / \mathrm{ml} 3$ horas após administração. Considerando os resultados do modelamento PK/PD, a dose de $80 \mathrm{ug} / \mathrm{mL}$ via IM permite alcançar as concentrações plasmáticas suficientes para se obter efeito de analgesia desde os 5 min e até as 3 horas. O estudo de Mama et al. (2009), demonstrou que a dose de 30 $\mathrm{ug} / \mathrm{kg}$ via IV foi suficiente para alcançar a concentração de $25 \mathrm{ng} / \mathrm{mL}$, necessária para máximo efeito antinociceptivo durante um período de 35 a 40 minutos. Enquanto a dose $80 \mathrm{ug} / \mathrm{kg}$ via IM permitiu atingir concentrações necessárias para obter o efeito analgésico máximo desde aproximadamente os cinco minutos e por um período em torno de três horas.

O efeito antinociceptivo simulado a partir das concentrações plasmáticas de correspondentes a doses de $80 \mathrm{ug} / \mathrm{kg}$ foi de $90 \%, 80 \%$ e $40 \%$ até 120,180 e 240 minutos para a via IM (FIGURA 5) e foi de $90 \%, 80 \%$ e $60 \%$ até, 120, 180 e 240 minutos para a via IV, respectivamente (FIGURA 6). 


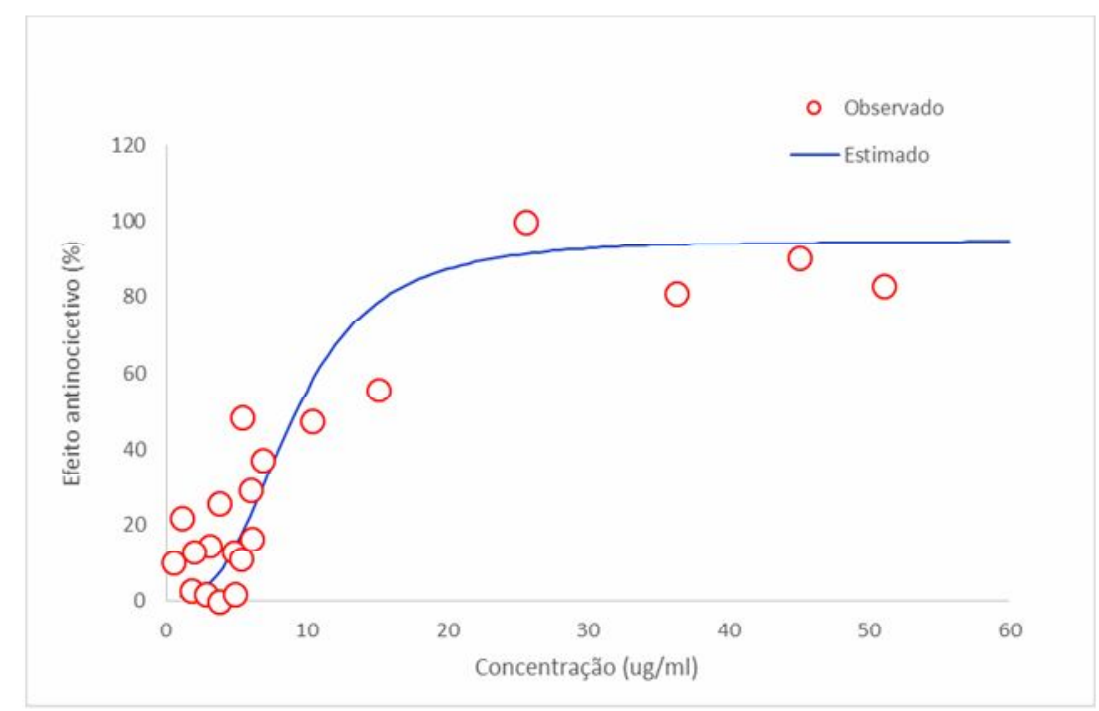

FIGURA 4. Analises PK/PD do efeito antinociceptivo de DET e em cavalos.

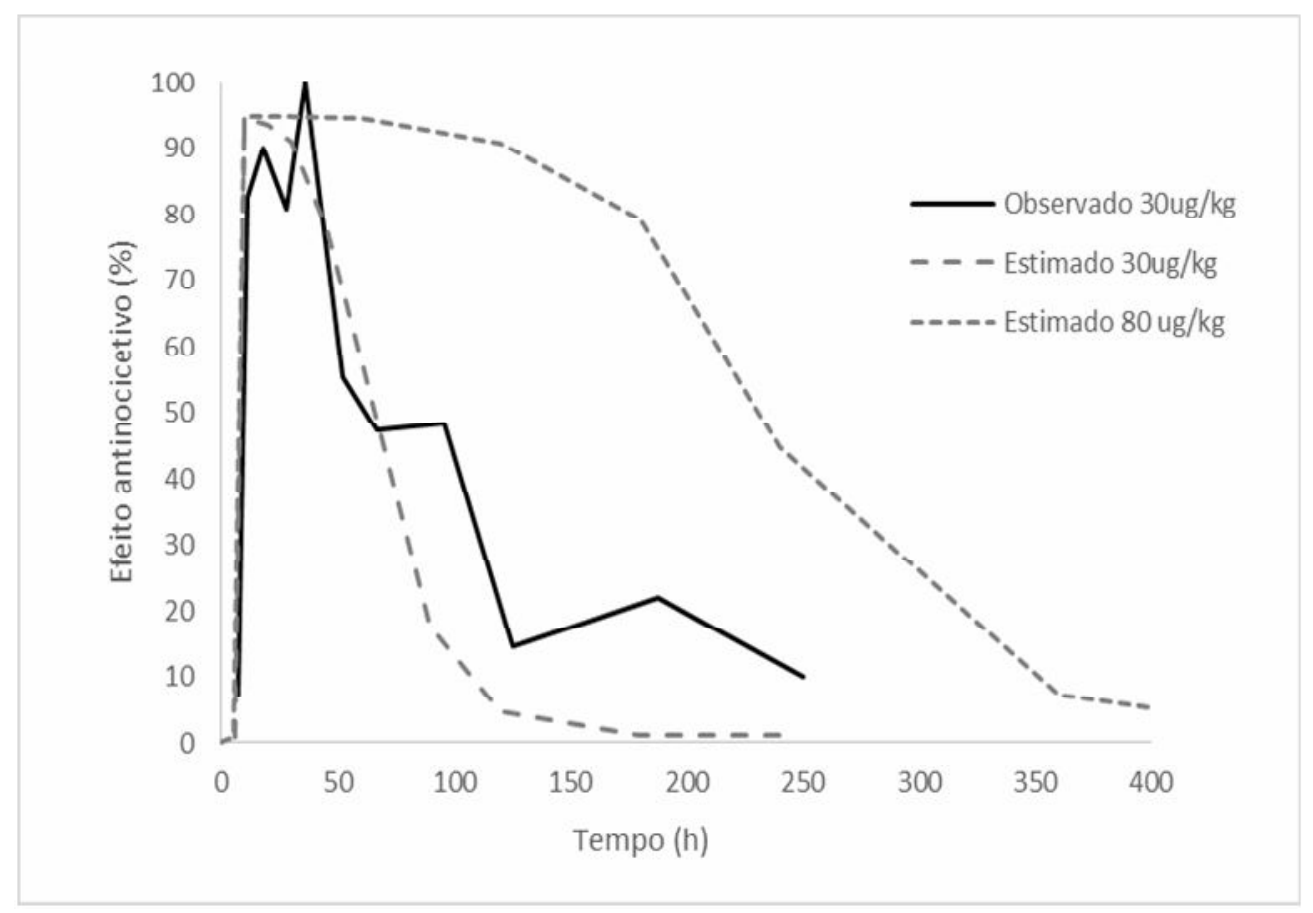

FIGURA 5. Efeito antinociceptivos de DET por via intravenosa em cavalos. 


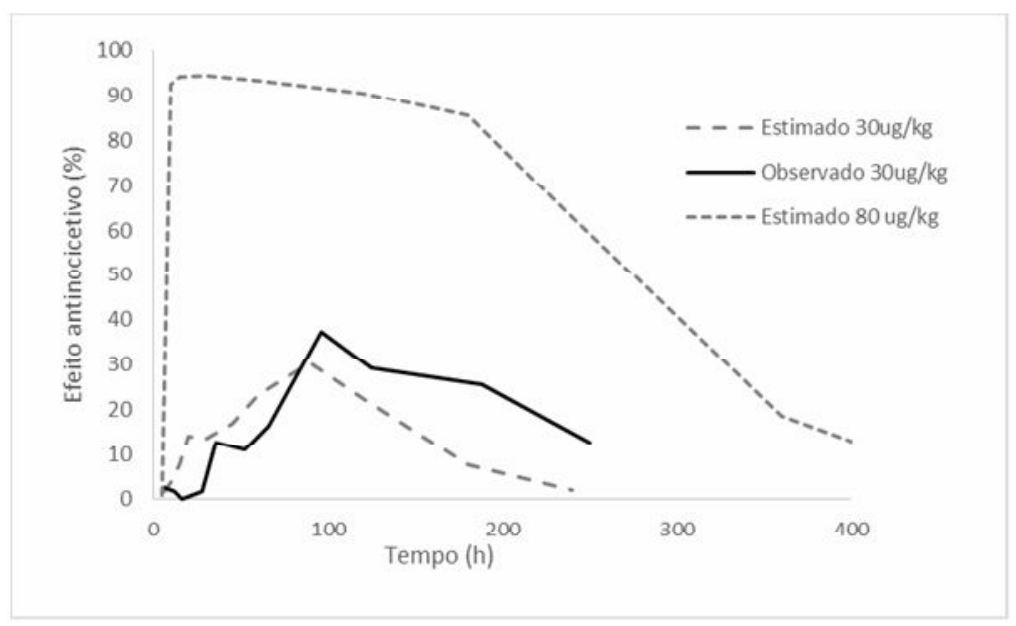

FIGURA 6. Efeito antinociceptivos de DET por via intramuscular em cavalos.

\section{DISCUSSÃO}

Love et al. (2012) avaliaram simultaneamente farmacocinética plasmática de buprenorfina e os efeitos antinociceptivos em cavalos. Seguidamente, a partir do analises PK/PD destes dados foi possível estimar os efeitos antinociceptivos com diferentes doses de bupremorfina mediante simulações (Love et al., 2015). De modo similar, esta analises PK/PD, permitiu utilizar a informação da dispocição plasmática com a doses de $80 \mathrm{ug} / \mathrm{kg}$ de DET para estimar o efeito antinociceptivo.

$O$ modelamento PK/PD realizado permitiu identificada que para atingir o efeito antinociceptivo adequao são necessários concentrações plasmáticas próximas a $20 \mathrm{ng} / \mathrm{ml}$, por tanto, com este resultado é possível realizar os cálculos de doses de infusão contínua para manter um efeito antinociceptivo por tempos prolongados.

Por outro lado, os resultados do presente estudo estimaram o tempo e a magnitude do efeito analgésico das doses de $80 \mathrm{ug} / \mathrm{kg}$ de DET por via IV e IM indicando a possibilidade da utilização destas doses para processos dolorosos de maior duração como por exemplo para cavalos com dor abdominal notória que não são candidatos para cirurgia exploratória ou que deve ser transportou grandes distâncias para intervenção cirúrgico.

Os fármacos alfa-2 Agonistas além do efeito analgésico e sedativo eficaz, também produzem outras alterações fisiológicas dependentes da concentração plasmática como ataxia, bradicardia, arritmias, aumento da resistência vascular sistêmica e redução do débito cardíaco (GONZALLO- MARCILLA et al., 2017). Neste sentido, a traves da comparação das concentrações plasmáticas máximas (FIGURA 3) e possível inferir que a dose de $80 \mathrm{ug} / \mathrm{kg}$ pela via IM determinaria efeitos adverso menores que a administração por via IV da doses de $30 \mathrm{ug} / \mathrm{kg}$ e que a administração da doses de $80 \mathrm{ug} / \mathrm{kg}$ por via IV poderia determinar efeitos adversos superiores à mesma. Desta forma, a dose de $80 \mathrm{ug} / \mathrm{kg}$ administrada pela via IM seria a mais adequada para produzir efeito analgésico mais prolongado. Porém, existe a necessidade da realização de estudos complementares de segurança para a posterior utilização das doses de $80 \mathrm{ug} / \mathrm{ml}$ na pratica clínica equídea.

\section{CONCLUSÃO}

O estudo permitiu estabelecer que a administração de doses de DET de $80 \mathrm{ug} / \mathrm{kg}$ provocam efeitos superiores e mais 
douradores que as doses de $30 \mathrm{ug} / \mathrm{kg}$ pelas respectivas vias de administração. Porém, são necessários realizar estudos de in vivo completares nessas doses elevadas que verifiquem os efeitos adversos.

\section{REFERÊNCIAS}

BILLARD, V. Pharmacokinetic- pharmacodynamic relationship of anesthetic drugs: from modeling to clinical use. F1000 research, 2015. Disponível em: < https://www.ncbi.nlm.nih.gov/pmc/articles/PMC4754004/>.

doi: 10.12688/f1000research.6601.1

ELFENBEIN, J. R.; ROBERTSON, S. A.; MACKAY, R. J.; KUKANICH, B.; SANCHEZ, L. C. BMC Veterinary Research, v. 10, n. 1, 2014. Disponível em: < https://www.ncbi.nlm.nih.gov/pmc/articles/PMC4123056/>. doi: 10.1186/1746-614810-S1-S6

GONZALLO-MARCILLA, M.; OLIVEIRA, A. R.; FONSECA, M. W.; POSSEBON, F. S.; PELLIGAND, L.; TAYLOR, P. M.; LUNA, S. P. L. Sedative and antinociceptive effects of different detomidine constant rate infusions, with or without methadone in standing horses. Equine Veterinary Journal, 2018b. Disponível em: < https://onlinelibrary.wiley.com/doi/full/10.1111/evj.13054>.

https://doi.org/10.1111/evj.13054

GONZALO-MARCILLA, M.; LUNA, S. P. L.; CROSIGNANI, N.; FILHO, J. N. P. P.; POSSEBON, F. S.; PELLIGAND, L.; TAYLOR, P. M. Sedative and antinociceptive effects of different combinations of detomidine and methadone in standing horses. Veterinary Anaesthesia and Analgesia, v.44, n. 5, p. 1116-1127, 2017. Disponível em: < https://www.vaajournal.org/article/S1467-2987(17)30174-5/fulltext> doi: https://doi.org/10.1016/j.vaa.2017.03.009

GONZALO-MARCILLA, M.; LUNA, S. P. L.; MOREIRA AS SILVA, R.; CROSIGNANI, N.; LOPES, N. P.; TAYLOR, P. M.; PELLIGAND, L. Characterisation of the in vivo interactions between detomidine and methadone in horses: Pharmacokinetic and pharmacodynamic modelling. Equine Veterinary Journal, 2018a. Disponível em: < https://onlinelibrary.wiley.com/doi/full/10.1111/evj.13031>. https://doi.org/10.1111/evj.13031

JONES, H. M.; MAYAWALA, K.; POULIN, P. Dose Selection Based on Physiologically Based Pharmacokinetic (PBPK) Approaches. The American Association of Pharmaceutical Scientists Journal, v.15, n. 2, p.377-387, 2013. Disponível em: < https://www.ncbi.nlm.nih.gov/pmc/articles/PMC3675752/>. doi: 10.1208/s12248-012-9446-2

LOPES, C.; LUNA, S. P. L.; ROSA, A. C.; QUARTERONE, C.; CROSIGNANI, N.; TAYLOR, P. M.; PANJOLA, J. C.; PUOLI, J. N. P. Antinociceptive effects of methadone combined with detomidine or acepromazine in horses. Equine Veterinary Journal, v. 48, n. 5, p. 613-618, 2017. Disponível em: $<$ https://onlinelibrary. wiley.com/doi/full/10.1111/evj.12483>.

doi: https://doi.org/10.1111/evj.12483

LOVE, E. J., TAYLOR, P. M., MURRELL, J. WHAY, H. R. Effects of acepromazine, butorphanol and buprenorphine on thermal and 
mechanical nociceptive thresholds in horses. Equine Veterinary Journal, v.44, n.2, p.221-225, 2012. Disponível em: https://onlinelibrary.wiley.com/doi/full/10.1111/j.2042-3306.2011.00412.x>. doi: https://doi.org/10.1111/j.2042-3306.2011.00412.x

LOVE, E. J.; LUDOVIC PELLIGAND, L.; POLLY, M.; TAYLOR, T. M.; JOANNA, C., MURRELL, J. C.; JOHN, W; SEAR, J. W. Pharmacokinetic- pharmacodynamic modelling of intravenous buprenorphine in conscious horses. Veterinary Anaesthesia and Analgesia, v.42, p.17-29, 2015. Diposnível em: < https://www.sciencedirect.com/science/article/abs/pii/S1467298716301209?via\%3Di hub>. doi: https://doi.org/10.1111/vaa.12165

MAMA, K, R.; GRIMSRUD, K.; SNELL, T.; STANLEY, S. Plasma concentrations, behavioral and physiological effects following intravenous and intramuscular detomidine in horses. Equine Veterinary Journal, v. 41, n. 8, p.772-777, 2009. Disponível em: < https://www.ncbi.nlm.nih.gov/pubmed/20095225>.

RANKIN, D. C. Sedativos e Tranquilizantes. In: GRIMM, K. A.; LAMONT, L. A.; TRANQUILLI, W. J.; GREENE, S. A.; ROBERTSON, S. A. Lumb \& Jones Anestesiologia e Analgesia em Veterinária. Rio de Janeiro: Guanabara Koogan, 5 ed. 2017. p.188-198.

SALONEN, J. S., VAHA-VAHE, T., VAINIO, O.; VAKKURI. O. Single-dose pharmacokinetics of detomidine in the horse and cow. Journal of Veterinary Pharmacology and Therapeutics, v.12:, p.65-72, 1989. Disponível em: < https://onlinelibrary.wiley.com/doi/abs/10.1111/j.1365-

2885.1989.tb00643.x?sid=nlm\%3Apubmed>. doi: https://doi.org/10.1111/j.13652885.1989.tb00643.x 